\title{
A Study on the Development Plans of Smart Phone Game Industry
}

\author{
Seok-Woo Shin ${ }^{1}$, Sang-Hyeon Park ${ }^{2}$, Yang-Woo Park ${ }^{3}$ and Seung-Il Moon ${ }^{4}$ \\ ${ }^{1}$ Lunosoft Inc., Seoul, Korea \\ xiyu@lunosoft.co.kr \\ ${ }^{2}$ Hanyang Cyber University, Seoul, Korea \\ anytime365@naver.com(CorrespondingAuthor) \\ ${ }^{3}$ Chung-Ang University, Seoul, Korea \\ ywpark1010@cau.ac.kr \\ ${ }^{4}$ Hanyang University, Seoul, Korea \\ moonsi921@hanyang.ac.kr
}

\begin{abstract}
The advent of the smart phone enjoying the web with a mobile phone and App store that is a mobile contents open market beyond the closed environment of the existing mobile carrier give many mobile game developers opportunities and risis at the same time. In a situation undergoing a lot of changes in this mobile nvironment, measures investigated through stakeholder interviews about mobile game,in ustry's development plans are as follows:

First, national policy support is required Second, developer's recognition on changes in the market is required. Third, the development of new business models such as partial charging, etc is required.
\end{abstract}

Keywords: Mobile Web 2.0, Game Industry, Mobile Game, Mobile contents

\section{Introduction}

In the mobile sector, many changes such as the advent of the smart phone and changes in the wireless internet environment such as Wi-Fi, LTE, the appearance of the mobile contents open market such as Apple's App store and Google's Android Market, etc are happening [1]. With the advent of the smat phone such as Samsung's T-Omnia phone or Apple's iPhone, Full Browsing that people ean see wired internet sites freely with mobile phones has emerged as a new killer app of mobile communication [2]. In 2008, Apple registered 25,000 applications unti1 January, 2009, expected sales of $\$ 1$ billion with 500 million of cumulative download and created new markets of mobile contents after opening App store 'for iPhone' that is a mobile contents open market rather than an existing carrier-oriented closed distribution network [3]. According to [4] App store application survey, applications that ranked one hundredth for one month, December 2008 are a total of 142, 62.4\% of them is games so that the field of mobile games is absolutely popular.

The mobile game's growth is expected to sustain because the rapid development of the network environment, multimedia services provided and high-tech handsets emergence in domestic mobile contents market environment $[5,6]$. Noted that so far hardware upgrades and appearance design-oriented differentiation are core values of the mobile phone, in future optimization of contents service in mobile handsets will take place it. Mobile carriers have also concentrated how to activate it, portal enterprises have atmosphere to worry about how to react contents as a key driver of future business rather than the level of preparing just the future, and contents suitable for it can be seen mobile games. 
In the era of Mobile Web 1.0 of WAP-based wireless internet environment in the past, people were able to access contents through the browser of mobile carriers themselves such as LG Telecom's ez-i and SK Telecom's Nate because domestic wireless internet service was closed. To do that, the frequency of the web use was insignificant. However, in the era of Mobile Web 2.0 established with the advent of the smart phone, open services were available, users involved directly and led changes [7, 8]. In addition, it's noted that Wi-Fi access feature is equipped in the smart phone and the mobile game will develop into the network game with large amounts or service web browser games by taking advantage of its fast speed and free fee [9].

There are not a lot of researches related to mobile games compared to changes in this mobile environment. Until now, the study on mobile games remains to the extent of mobile games usage status. Therefore, the purpose of this study is to analyze what is required to develop the mobile game industry and to propose ways on this in the era of Mobile Web 2.0. It's expected that the results of this study are useful in mobile game policy establishment and development.

\section{Theoretical Reviews}

\subsection{Characteristics of Mobile Web 2.0}

The most distinctive feature between mobile Web 1.0 and 20 is to transfer from closed model to open model in business environment. In mobile web 1.0, the carrier operates its own network and customers can use limited contents that it supplies and they can't change mobile phone standby screen itself. On the other hand, Mobile web 2.0 is possible to implement wired and wireless integrated environnentas an open model. The interface similar to PC can be implemented because it's possible to provide various Mashes up services through open API and to consist of user-centered menu [10].

Looking at mobile web 2.0 features, first Mobile web as a platform' combining a wide range of mobile technologies and applications is possible [11]. Second, Mash up is available as the standard such as XML and Mobile OK that are its standard and openness features is created and Open APT is serviced. Third, constraints of mobile devices are minimized and its mobility's advantages are maximzed by utilizing the network with various transport levels such as WiFi and Wibro and user interface based on the technology for ubiquitous web access. Fourth, applications and serve models which search and ads that is possible only in the wired are associated with are common, business models considering the long-tail rather than traditional top $20 \%$ of users have occurred. Fifth, closed mobile carrier-oriented service environment in the past became to be changed into open user-oriented service environment [12].

Thus mobile web 2.0 will be an upgraded core platform that makes users identify information on various objects and environment conveniently with certainly more automated enviroment and that plays an important intermediary role connecting personal life and community, information space [8].

As shown in Yahoo Go! Mobile, Google Mobile services, a variety of attempts for connecting search and existing services have been attempted. Nokia has promoted the development of the technology to combine mobile games and ads. As supporting this, in ABI research forecasting data(2007) on mobile marketing and advertising market's scale, it's noted that its scale is $\$ 3$ billion in 2007 and its scale will \$ 19 billion including mobile research and video advertising market.

One of application technology trends that are most popular recently can be SNS(Social Network Service). The interest on social networking technologies in the field of mobile is 
growing by increasing Korea's Cyworld, U.S's My Space services, etc and presenting various techniques about social bookmarking and social networking. It's noted that in this background, the possibility of mobile social network can be large in that it has been utilized as a means for mobile device characteristics and communication with others as a personal terminal to keep important personal information (ABI Research, 2007). The growth of EZ GREE, Mixi, Mobage Town in Japan and (UGC:User Generated Contents) technology's combination have been a momentum to show various application possibilities of mobile social networking technology.

Table 1. Compared Mobile Web1.0 with Mobile Web 2.0

\begin{tabular}{|c|c|c|}
\hline & Mobile Web 1.0 & Mobile Web 2.0 \\
\hline Network & low speed $(<0.5 \mathrm{MB})$ & high speed(>0.5MB)-HSDPA, WiBro \\
\hline Protocol & WAP protocol-based WAP browsing & (w)TCP/IP-based full browsing \\
\hline Contents & HTML\&WML-centered contents & XML\&XTHM -centered contents \\
\hline $\begin{array}{l}\text { Business } \\
\text { model }\end{array}$ & closed business model & $\begin{array}{l}\text { lel, wired and wireless } \\
\text { tion model }\end{array}$ \\
\hline $\begin{array}{l}\text { Technology } \\
\text { model }\end{array}$ & closed, independent & \\
\hline $\begin{array}{l}\text { Browsing } \\
\text { method }\end{array}$ & & $\begin{array}{l}\text { Browsing linked with RFID and } \\
\text { BS, RSS reader function }\end{array}$ \\
\hline Device & & various mobile devices \\
\hline Service & & $\begin{array}{c}\text { REST, SOAP, WSDL -based mobile web } \\
\text { services }\end{array}$ \\
\hline Certification & & distributed certification, Identity Management \\
\hline Access & by hand & $\begin{array}{l}\text { automatic access method(WINC, mobile } \\
\text { RFID, 2D barcode, etc) }\end{array}$ \\
\hline UI & Mands/hands free & $\begin{array}{l}\text { multi-modal/ubiquitous web access } \\
\text { technology(voice, gestures, RFID etc) }\end{array}$ \\
\hline API linkage & one service and part of API & open API and Mashup services \\
\hline Charge & -rate system(high cost) & flat sum system based(low cost) \\
\hline Ads & no ads & new business model based on mobile ads \\
\hline Features & browsing only & mobile web as a platform \\
\hline
\end{tabular}

Mobile contents usage is convenient due to the development of mobile communication technology and various contents such as ring tones, call-waiting tone, and mobile games have been serviced. Domestic mobile game market has been a high growth in recent years through service users' surging trend, the rapid development of technology, recent high-end devices' major widespread atmosphere, in the future, the improvement of the mobile game industry is expected to continue [12]. 
In the early stages, mobile games were mainly classic games familiar to consumers rather than creative games due to the limited platform, and puzzles to operate simply but in the current mobile game market, it displays 3D games or MMORPG games, various games equipped with the high quality beyond portable game consoles in the past. In addition, it's expanding the area with creative mobile games combined with movies, music, broadcast contents [13].

\section{Practical Survey}

\subsection{Research Methods and Characteristics of Respondents}

In order to achieve the purpose of this study, researchers tried to derive development plans by dong in-depth interviews with experts in the field of mobile ie mobile ganne representatives of the carrier, marketing and development managers in leading mobile game companies and persons in charge of mobile game community. Researchers made respondents identify the contents of questions and think the answer in adyance and then represent their position, opinion, feeling, by conducting semi-standardized interview of In-depth interview targeting professionals. Those who were doing the work assaciated with mobile games and have at least 3 years were selected as interviewees. The reason why this researcher has these criteria is that three years is the period that their own opinions related to mobile games are reflected in the company or they have their own thinking, and that they should be working to consider recently rapid changing mobile environment andomobile game's status and development plans.

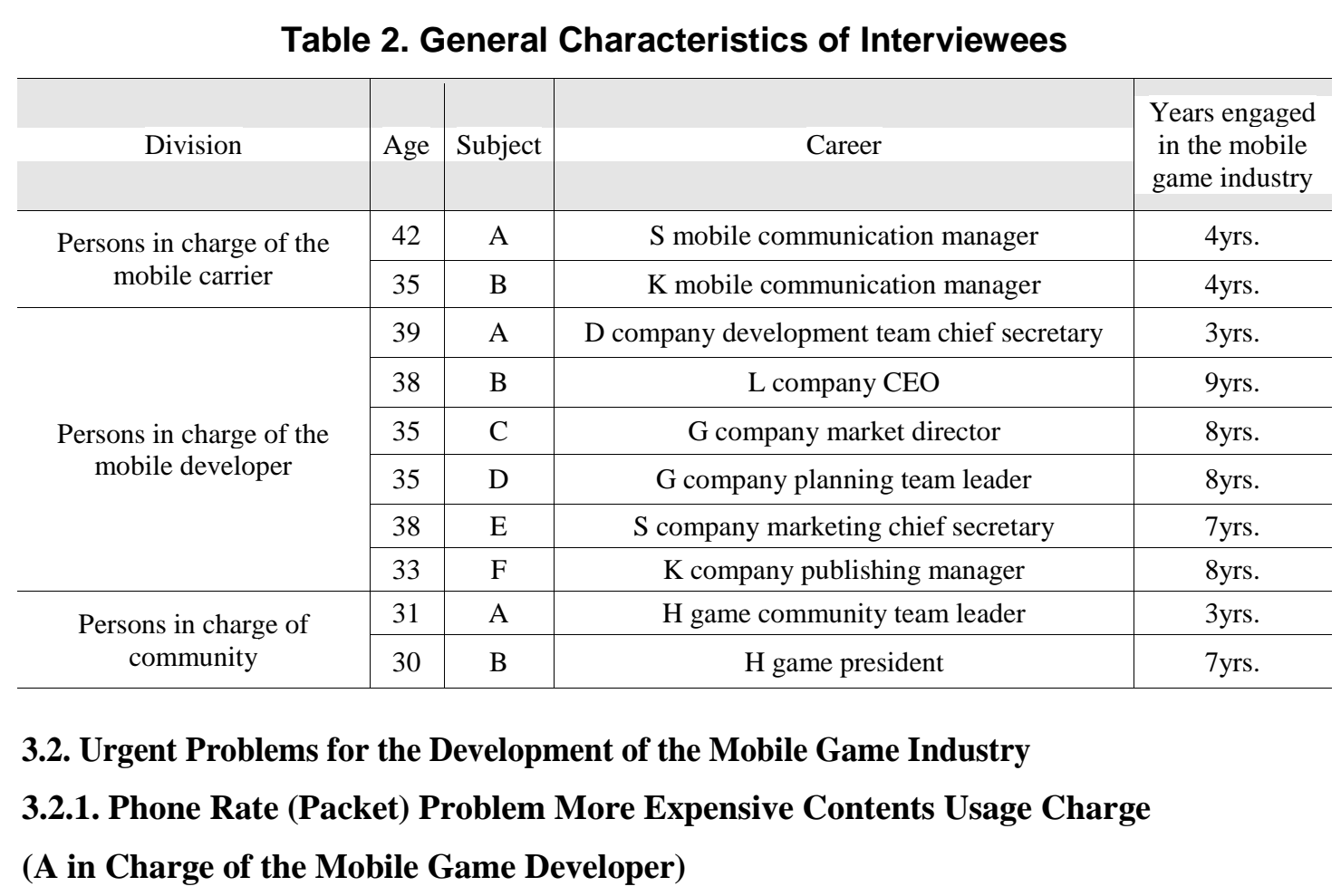

It's thought that if mobile phone rte and internet rate should be cut a little for users, additional purchasing power on the contents is higher, if open net is more activated, exposure is strengthened and billing/download server is carried out, it leads to substantial net open and through this, various industries shall be formed. It's important to change to user-centered 
structure without power competence between carriers and devices manufactures such as WIFI equipment limit and DRM removal, etc.,.

\subsubsection{Uniform Game Sales Approach Dependent on Carriers}

\section{(B in Charge of the Mobile Game Developer)}

Sales Flow that serves games is too undifferentiated. Through mobile carriers' mobile portal, most of the game developers and publishers stick to the work and actual game purchasing also is done. It's absolutely necessary that this structure is changed opening mobile 2.0 market but it doesn't seem to be easy when thinking hegemony of the carriers. The reason is that even handset market that is a bigger market release various specifications(smart phones, luxury phones) but the success of the phone depends significantly on carriers

\subsubsection{Etc.,.}

\section{( $C$ in Charger of the Mobile Game Developer)}

After abolishing WIPI obligations installed in mobile phones releasing on April 2009, unified specification of the hardware and development language don't exist. Smart phones equipped with various types of OS such as Android, Window mobile and Linux, come into the domestic, there are few hose that can develop mobile game in such mobile phones with OS in Korea.

When asking urgent problems for the derepment of the mobile game industry, it was thought to answer only expensive packet rate Of course, prost people said that the problem of phone rate (packet rate)more expensive than game contents usage charge, in addition there were a uniform game sales methodand the dommance of mobile carriers (of course, the problem of costly packet rate is a part of the dominance of mobile carriers) and diversified development environment, and those who operate companies commented unexpected revenue structure and government support. A story regarding win-win fund comes in the recent $S$ carrier, though carriers support mobile game developers, industry executives told the support for mobile game developers is too weak in carriers that take whole sales of packet rate and $10 \%$ of contents usage charge revenue.

\subsection{Status of the Mobile Game Industry}

\subsubsection{The Limitations of the Game for Killing Time (D in Charge of the Mobile Game Developer)}

Yet it seems to play a role of niche markets (portable) of online, game console. Exactly, it seems to be used for coming up for such as the toilet, commuter, etc. In recent years, although the levels of mobile RPG games and users who enjoy at home and school have increased, those of o her genres don't seem to play a role beyond killing time.

\subsubsection{Mobile Game as one Source Multi-use Usage}

\section{( $E$ in Charge of the Mobile Game Developer)}

The mobile game industry is growing in terms of popular package game or one source multi-use usage on line, it has a limitation to obtain popularity in some genres of games such as RPG, Tycoon rather than various genres of games. It's necessary to emerge new genres suitable for changes of devices and users.

Answers intended in questions of how the mobile game industry's position is were slightly 
different. It was noted that compared to most online games, the total sales of the mobile game industry was less compared to NC Soft which reached 350 billion won in 2008, despite of calling the mobile game industry its overall sales was less. It was a general opinion that a role as killing time purpose and one source multi-use that is a game succeeded with other platform unlike online games was mentioned so that the mobile game was lack of mentioning as its own industry. Most of them thought that although independent mobile game industry association had been established in the end of 2004, it became absorbed in Game industry association focusing on online games, the effect of mobile carries unlike other industries is absolute so that the mobile game was hard to be recognized as one industry. However, they thought that iPhone was emerged, mobile contents open market was activated, so if the influence of mobile carries was decreased, the appearance of fixed packet rate was escaped, Korean mobile game would have the appearance of independent industry sooner or later.

\subsection{Policy Needed in the Mobile Game Industry}

\subsubsection{Various Support Policies for the Mobile Game Industry ( $F$ in Charge of the Mobile Game Developer)}

It's thought that there are various plans to foster intimidated incentives such as game development support, etc in the short term, after all in the long tern, it's necessary to have the policy to induce constantly the development of the practical net and transition to web 2.0 environment through multiple operators application such as the fee for wireless internet cuts, WI-FI development, net rental/billing/download server. If so, it's to be better by emphasizing advantages of the mobile game industry with the activation of the network game.

\subsection{Opinions on Activating the Mobile Game Industry}

\subsubsection{Unified Game Platform Needed}

\section{( $G$ in Charge of the MobileGame Developer)}

For a while, network games and semi-network games, etc were hot in the entire mobile games but eventually they were not activated due to expensive packet rate. Partial charging appeared from the second half of 2007 is a trend now, some RPG games are shown that partial charging sales are highe than contents usage charge sales. It's thought that more partial charging models vill emerge in the future and mobile games like online games need revenue channels expansion through advertising within the game. If packet rate is adjusted realistically, rate system modification and net openness are plans to activate the mobile game so those customers who want network games again may enjoy them in more affordable price.

\section{Improvement Plans of the Mobile Game Industry}

In this study, three measures were suggested to activate mobile web 2.0 based-mobile game industry, which could be summarized as follows:

\subsection{National Policy Support Needed}

$\mathrm{B}, \mathrm{H}, \mathrm{Yu}$ who is a manager of cultural contents industry in the Ministry of culture, sports and tourism noted he would pursue a active support policy to grow the game industry as the core contents industry in the future. In addition [14], receiving attention recently noted that a new national aid policy on new forms of distribution such as the mobile contents open market was needed. 


\subsection{Mobile Instant Messenger Game Service Involved}

As MIM (Mobile Instant Messenger) is emerging as a core platform to replace the portal in the mobile era, competition surrounding this is becoming increasingly fierce. Strategy Analytics, market research Company in the U.S (2013) expected the carrier would lose over $\$ 3$ billion revenue for 6 years since 2012 with messenger services such as Kakao Talk, Line, and Eye messenger.

\subsection{Developer's Recognition on Changes in the Market}

Gamevil followed by Apple's App store, Google's Android market, Rim's Blackberry App world, Samsung's Application store, preoccupied already global open markets, 'Zenonia' was exposed to 'Showcase' menu that selects excellent applications in Microsoft part carefully and shows them so that it has a bright prospect. It's the only company in the industry to establish the local subsidiary in the U.S. and plans to put a large portion by targeting the overseas markets before activating the open market and keeping pace with the growth of the open market being expanded to the world.

\subsection{Development of New Business Models such as Dartial Charging, ete., Needed}

The mobile game industry has entered a new growth phase due to wireless internet infrastructure expansion and various billing models application. In the past, as the online game industry grew rapidly due to internet flat rate and its infrastructure expansion, the mobile game industry shall be similar.

\section{Conclusion}

It has already been 14 years since the dornestic mobile game market was formed. The domestic and international market environment is going to change into mobile web 2.0 environment. Among them the advent of the smart phone to enjoy the web with the mobile phone and App store than is a mobile contents open market gave the mobile game developers opportunities and crises at the same time.

Therefore, the purpose of this study was first to identify the rapidly changing mobile environment with the impact of the mobile web 2.0, second to examine improvement plans in the domestic moble game incustry developing with changes in the mobile environment.

This study was to investigate changes of the mobile phone device environment and the mobile game industry undergoing a big change recently in the same purpose as above. This researcher conducted expert interviews due to the lack of previous research related to the mobile game industry. Mobile web 2.0 environment and ways to activate the mobile game industry

Through nterview for about 1 hour by visiting the interviewees such as persons in charge of the mobile carrier, the mobile game developer and the mobile game community could be found

As a result of the empirical analysis, first of all, this research could understand the mobile web 2.0 environment.

First, the most distinguishing feature in the mobile web 2.0 is that the business environment changes from the closed model to the open model. Second, it's possible for consumers to have technical support for wireless internet access so that they may use data calls rate free or nearly free by utilizing the network with various transport layers of Wi-Fi and WiBro. Third, the closed business model in the form of Walled Garden served focusing on some carriers is weaken, consumers can buy contents which they want through App store 
that is a mobile contents open market.

Thus, in the situation undergoing a lot of changes in the mobile environment, measures identified through interviews with stakeholders regarding development plans of the mobile game industry are follows:

First, national policy support is required. Regarding things small mobile game developers don't do directly with larger carriers, national policy support is needed. Second, developers' recognition on changes in the market about the rapidly changing mobile environment is required. Third, it's necessary to develop new business models such as partial charging, etc., the life cycle of the domestic mobile game was 3 months before but that of some games is has a long run of more than 1 year through this partial charging.

This study was to do focusing on the contents of activating the mobile game industry based on mobile web 2.0 which hadn't been addressed intensively by this time. Based on this, it's expected that the domestic mobile game industry corresponds positively several environmental changes and research on measures of the mobile game industry shall be steadily done. In particular, research activities and practices in detail about measures suggested for activating the mobile game industry should be done.

\section{Reference}

[1] B. Y. Jeong, "Mobile Game Status and Opportunity by Market participants", Information Communication Policy, vol. 16, no. 17, Serial no. 355, (2004), pp. 19 20

[2] J. E. Yu, "Smartphone's Key Enabler: Software", KIPA SW insight Poliey Report, no. 42, (2009), pp. 26-29.

[3] J. I. Kwon, "Domestic and International Mobile application market status", Policy of Broadcasting and Telecommunication, vol. 21, no. 7, Serial no. 460, (2009).

[4] S. I. Lee, "Mobile internet business strategies CfMobile Carners? ITA, Weekly Technical Trends, Serial no. 1388, (2009), pp. 14 17.

[5] H. G. Park, "A study on Mobile Game Status and Preference Analysis", Journal of design culture, vol. 4, Serial no. 10, (2004).

[6] S. C. H. Jeong, "Recent trend on Moblle business", GBusiness Insight, (2008) March 12, pp. 19-21.

[7] Y. G. Kim, "A topic IPhone-brought up Mobile Communication Market", Weekly LG Economic, (2007) August 1.

[8] J. H. Jeong and S. Y Lee, Mobile Web 2.0 and Mobile OK Standardization Trend", A Trend Analysis on Electronics and Telecommunication, vol. 22, no. 6, (2007).

[9] J. H. Park, "Coping Strategies and Implications of Mobile Carriers depending on the Introduction of Application Stores, Policy of Broadeasting and Telecommunication, vol. 21, no. 9, Serial no. 462, (2009), pp. $2 \sim 5$.

[10] H. J. Kang, "Changing Market Condition and Prospects in the era of Mobile Web", Samsung Economic Research, Issue Paper, (2008) March 11.

[11] J. H. Jeong and S. Y. LEF, "Mobile Web 2.0 Technology Prospects", Telecommunications Review, ETRI, vol. 17, no. 4, (2007) August, pp. 629-630.

[12] J. H. Park, "Coping Strategies and Implications of Mobile Carriers depending on the Introduction of Application Stores", Policy of Broadcasting and Telecommunication, vol. 21, no. 9, Serial no. 462, (2009), pp. $2 \sim 5$.

[13] "Korea game industry Promotion Institute", Korea Game White Paper, (2008).

[14] S. Lee, Mobile internet business strategies of Mobile Carriers", IITA, Weekly Technical Trends, Serial no. 1388,(2009). 


\section{Authors}

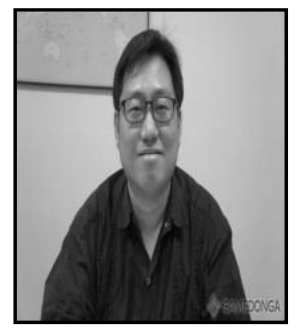

Seok-Woo Shin, he works as the department head of Game Studio Lunosoft Inc. and is in charge of the marketing and sales of the smartphone game. He is an expert in the mobile game industry with more than 10 years' experience. He graduated from the Entertainment Department in Hanyang University the Graduate School of International Tourism.

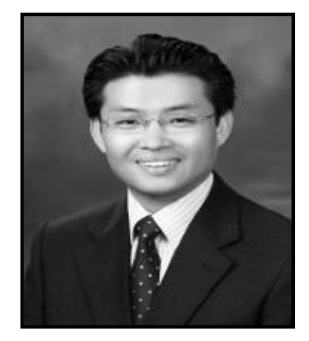

Sang-Hyeon Park, he is an associate professor of Hanyang Cyber University. He has a lot of interest in culture and entertainmentindustry. He has published many papers of these fields and given leetures for years. He received master's and doctor's degrees in Hanyang University.

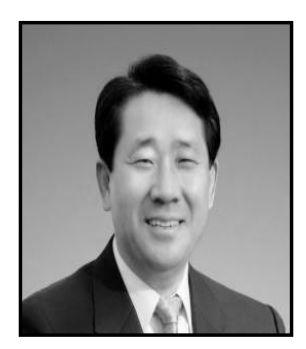

Yang-Woo Park, he is a professor of the Department of Art Management in Chung-Ang Unive sity. He is a former Vice-Minister of Culture, Sports and Tourism in Korea and former vice president of Chung-Ang Unyersity. He worked in the area of culture and art-related policy and administration 6ryore than 30 years. He received a doctor's degree in Hanyang University.

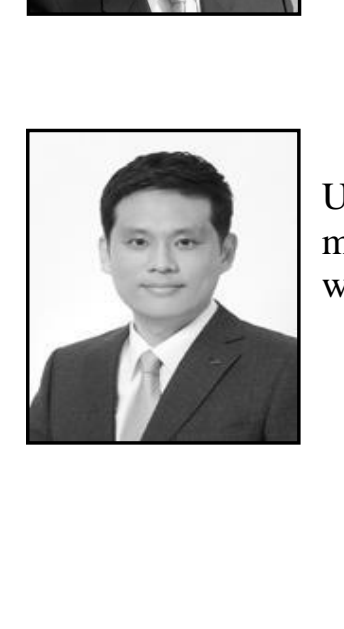

Seung-1I Moon, he is a professor of Hospitality Academy in Hanyang University $\mathrm{He}$ has a lot of interest in IT and information. He has written many papers and books on these fields and given lectures for years. He worked as a webmaster and Internet honorary police officer. 
International Journal of Multimedia and Ubiquitous Engineering Vol. 9, No. 12 (2014)

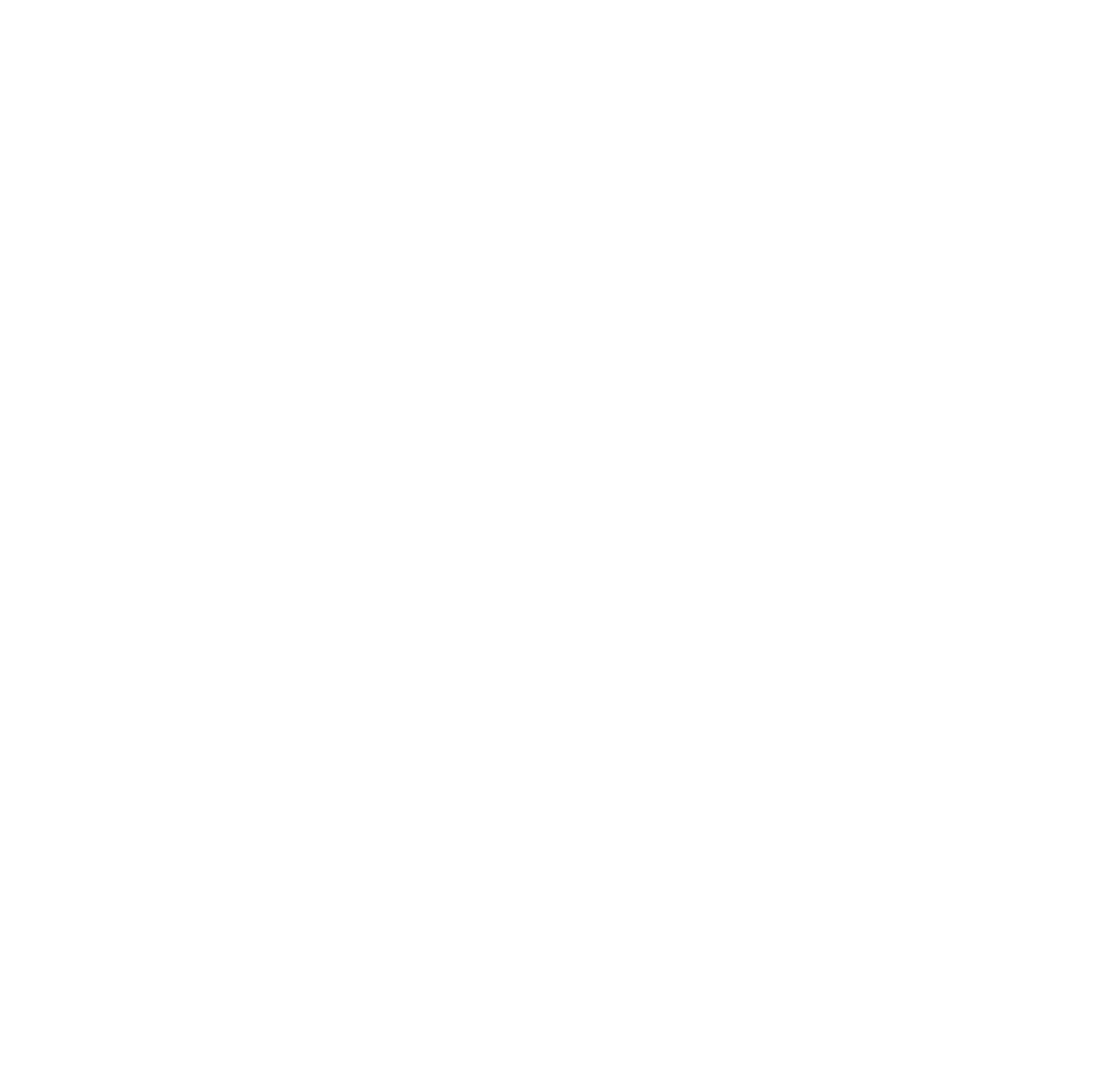

